\title{
Surfaces
}

\section{Dette, institution et histoire}

\section{Éric Méchoulan}

Volume 6, 1996

LES ÉCONOMIES DISCURSIVES DU SAVOIR ET DE LA CULTURE DANS LE SILLAGE DE L'OEUVRE DE BILL READINGS

THE DISCURSIVE ECONOMIES OF KNOWLEDGE AND CULTURE, WITH CONSTANT REFERENCE TO THE WORK OF BILL READINGS

URI : https://id.erudit.org/iderudit/1064859ar

DOI : https://doi.org/10.7202/1064859ar

Aller au sommaire du numéro

Éditeur(s)

Les Presses de l’Université de Montréal

ISSN

1188-2492 (imprimé)

1200-5320 (numérique)

Découvrir la revue

Citer cet article

Méchoulan, É. (1996). Dette, institution et histoire. Surfaces, 6. https://doi.org/10.7202/1064859ar
Résumé de l'article

Empruntant (sans les rendre immédiatement) quelques chemins de traverse (par exemple ce que c'est qu'une institution et ce qu'on lui doit dans des types de sociétés différents, comment fonctionne le discours historiographique et ce qu'il engage dans le rapport au passé et au présent, à l'altérité et aux morts), cette réflexion met en scène certains scénarios de la médiation et certains affolements face à l’immédiateté.
Copyright @ Éric Méchoulan, 1996

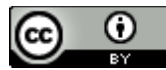

Ce document est protégé par la loi sur le droit d'auteur. L'utilisation des services d'Érudit (y compris la reproduction) est assujettie à sa politique d'utilisation que vous pouvez consulter en ligne.

https://apropos.erudit.org/fr/usagers/politique-dutilisation/ 


\section{Dette, institution et histoire}

Éric Méchoulan

Département d'Études françaises

Université de Montréal

mechoule@ere.umontreal.ca

Surfaces Vol. VI. 214 (v.1.0F - 12/10/1996) - ISSN:

1188-2492

Tout texte reste la propriété de son auteur. Néanmoins, Surfaces demande d'être citée à l'occasion de toute autre publication du texte en question.

\section{RÉSUMÉ}

Empruntant (sans les rendre immédiatement) quelques chemins de traverse (par exemple ce que c'est qu'une institution et ce qu'on lui doit dans des types de sociétés différents, comment fonctionne le discours historiographique et ce qu'il engage dans le rapport au passé et au présent, à l'altérité et aux morts), cette réflexion met en scène certains scénarios de la médiation et certains affolements face à l'immédiateté.

\section{ABSTRACT}

Skirting along certain worn pathways, without leaving them right away (for example, notions about what an institution is and what it is owed in different types of societies, about how historiographic discourse functions and what it entails vis-à-vis the past and the present, otherness, and the dead), this reflection plays out certain scenarios of mediation and certain turmoils in the face of immediacy. 
"Reading is not a matter of mimetic representation or conceptual critique: it is an ethical practice" Bill Readings, Introducing Lyotard:

Art and Politics, 1991.

"Criton, nous sommes le débiteur d'Asclépios pour un coq; eh bien! payez ma dette, pensez-y. - Bon! ce sera fait, dit Criton. Mais vois si tu n'as rien d'autre à dire. La question de Criton resta sans réponse. Au bout d'un petit moment, il eut pourtant un sursaut. L'homme alors le découvrit: son regard était fixe. Voyant cela, Criton lui ferma la bouche et les yeux[ $\underline{\mathbf{1}}$ ] . Serties dans une émouvante absence de pathos, telles furent les dernières paroles de Socrate, plutôt énigmatiques pour un tel amateur de clarté. Les savants ont bien sûr soigneusement cherché quel voeu avait bien pu faire Socrate pour suggérer semblable offrande, mais Léon Robin, avec grand bon sens, indique une signification symbolique évidente: Socrate sent que son âme est enfin guérie du mal d'être unie à un corps; sa gratitude va donc au Dieu qui rétablit la santé, Asclépios . Après ce grand discours sur l'immortalité de l'âme qu'est le Phédon, rien de plus logique en effet que de considérer la mort comme une nouvelle et plus parfaite santé. Mais on connaît le caractère retors de Socrate, ainsi que l'amitié bienveillante qu'il portait à ses compagnons en philosophie. Ne pourrait-on alors sonder cet ultime souhait au lieu de le laisser aller, comme sur son erre, vers les quais du symbole ou du référent? L'objet de la dette est peut-être moins crucial que le mouvement de la dette lui-même. Il importe moins en effet de connaître ce qu'il faut rendre en même temps que la vie, que de pouvoir reconnaître le fait même qu'il s'agisse d'une dette. S'il y a une certaine joie de mourir chez Socrate, c'est dans cette possibilité, à lui ainsi donnée, de reconnaître sa dette envers les dieux. Plus encore, faire payer sa dette par ses amis contribue à la fois à les inclure immédiatement dans cette reconnaissance de la dette, comme un ultime enseignement sur la valeur fondatrice des dettes, et à leur éviter tout sentiment d'endettement envers lui-même, Socrate, leur vieux maître et compagnon, puisqu'il leur abandonne le bonheur de régler ses dettes, autrement dit de servir de médiateurs entre lui et les dieux. 
Bill Readings, dans son attention portée aux institutions universitaires, nous rappelle une évidence trop facilement partagée : le fait qu'enseignement et recherche (la schizophrénie du professeur ordinaire) sont historiquement liés[ $\underline{2}$ ], comme si la production du savoir pouvait être réinvestie dans sa reproduction par l'enseignement, aussi facilement que le simple redoublement de la production dans le re-production semble l'impliquer. Or ici l'illusion régulatrice repose sur l'idée même de production .

Il n'y a d'économie - au sens classique du terme - que de la production. On ne peut faire l'économie de la production, on ne peut s'en passer. Et c'est en quoi on ne saurait faire une économie du don, car le don n'a aucun moyen d'être pensé dans une économie de la production. Plus exactement le don devient ce que l'économie (de la production) ne peut penser. C'est ce qui fait qu'un don, pour être parmi nous reçu comme don, ne doit jamais rien produire: ni gloire, ni honneur, ni capital symbolique pour le donateur, ni reconnaissance, ni contre-don pour le donataire. Comme est amené à le dire Derrida: le don n'est jamais présent, il est l'impossible - - tout au moins l'impossible dans une économie de la production, c'est-àdire dans l'économie occidentale moderne qui, en faisant tourner les planètes du social autour du soleil de la production, a donné au don et à la dépense une position spécifique en-dehors de ce système solaire. Du coup, de la même façon que nous rêvons d'échapper à notre environnement solaire, nous cherchons obstinément à gagner les régions, puisque étrangères, forcément plus éthérées et plus glorieuses du don. Comment cependant rendre raison du fait que l'on pourrait fausser compagnie à l'économie de la production? Mais, d'abord, qu'est-ce qui oblige à en rendre raison? Un principe, un principe très puissant puisqu'il est censé être le principe des principes: Leibniz est le premier à l'énoncer, c'est le principium reddendae rationis. Il y a, comme le souligne Heidegger, un appel à rendre raison dans ce principe. Or ce principe, Heidegger en montre le lien essentiel avec les temps modernes, avec donc une nouvelle manière de penser, ou plutôt d'oublier - en la formulant même - la question de l'être de l'étant.

À partir de la fin du XIVe siècle, dans les grandes cités marchandes d'Italie, se développe une nouvelle habitude chez les commerçants: celle de tenir des livres de compte, des mémoires où sont enregistrés les ventes et les achats, les acquis, les débits et les crédits, bref toutes les opérations financières de la famille. Mais ces livres de compte sont aussi écritures de contes, où se 
raconte l'histoire familiale, les événements marquants (non pas tant historiques, ceux qui concerneraient l'ensemble de la collectivité, que ceux des alentours familiaux), cela allant parfois jusqu'à une certaine réflexion sur soi, proche alors du journal tel qu'encore nous le connaissons. Ces registres que la famille se passe de génération en génération s'appellent en italien des Ricordanza, des mémoires (à la fois masculin et féminin pluriel). Avec le développement marchand de la Renaissance, la pratique se répand en Europe.

Cependant rapidement on ne les nomme plus mémoires, mais, de façon fort significative, livres de raison . Nom qui entre dans le dictionnaire de Furetière avec la définition suivante: Livre de raison est un livre dans lequel un bon mesnager ou un marchand escrit tout ce qu'il reçoit et despense pour se rendre compte et raison à luy mesme de toutes ses affaires . Il est donc devenu indispensable de se rendre compte de ce que l'on fait, mais aussi de se rendre compte de son moi et de se rendre des comptes à soi-même, à la fois donc réaliser ce que l'on est, pour mieux réaliser ce que l'on vaut. La raison, devenue affaire individuelle, est ce que l'on se rend à soi-même, ce qu'on est appelé à se rendre pour mieux en affirmer, au bout du compte, la souveraine possession: c'est une dette envers soi dont les livres de raison rendent compte en même temps que du profit dont le moi doit bénéficier (voire dont il figure la plusvalue). Et c'est peut-être dans ce court-circuit, ou dans cet aller-retour entre dette et profit que se réalise la puissance de la raison, la puissance de son appel à être rendue, de même que la puissance de l'individu à s'en voir investi et à la posséder. Mais s'il y a aller-retour entre dette et profit, la dette n'est jamais reconnue comme telle pour autant.

Le syntagme rendre la raison ne s'emploie pas sur le modèle de rendre un cadeau ou rendre la monnaie, plutôt sur celui de rendre la justice . Rendre raison suppose, comme y insiste Leibniz, le fait qu'il y a toujours quelqu'un qui peut la rendre. La dette implicite envers la raison s'est muée en pouvoir - ou plus précisément tout tient à une accentuation: la dette reste présente (sur le mode de l'oubli, de l'effacement) et tout le poids passe du côté du pouvoir à rendre raison.

L'avantage? cela permet enfin d'entendre rendre raison comme une production. D'un point de vue judiciaire, rendre la justice suppose en effet moins une exigence qui me vienne du dehors et qui m'appelle à être juste, que l'idée qu'il y a des jugements à produire pour décider qui a raison ou tort, et que ces jugements ne seront justes que s'ils sont produits par les personnes compétentes pour les porter. De même rendre raison 
met moins l'accent sur la dette qu'elle implique envers une altérité, un Autre qui nous appellerait à rendre raison, du dehors de nous-mêmes, que sur la possession qu'en bout de compte assure la raison: possession à la fois du moi qui a le pouvoir de rendre raison de toute proposition vraie, et possession de la raison elle-même qui qualifie le sujet capable de rendre compte de la raison.

Or cet aller-retour entre dette et possession, cet oubli de la dette et cet accent porté sur la possession, c'est aussi ce qui caractérise toute institution. Dans la partie intitulée Divagations d'un inactuel du Crépuscule des idoles, Nietzsche écrit: Nos institutions ne valent plus rien: là-dessus tout le monde est d'accord. Pourtant cela ne tient pas à elles, mais à nous. Une fois que nous avons perdu tous les instincts dont naissent les institutions, les institutions nous échappent à leur tour, parce que nous ne sommes plus dignes d'elles. (...) L'Occident tout entier a perdu ces instincts d'où naît un avenir : rien qui aille plus à rebours de son "esprit moderne". On vit au jour le jour, on vit très vite, on vit de manière très irresponsable: c'est précisément cela qu'on appelle "liberté". Ce qui fait que les institutions sont des institutions, on le méprise, on le déteste, on le repousse: on se croit menacé d'une nouvelle servitude dès que le mot autorité est prononcé à haute voix. On a bien trop vite fait de rejeter les institutions dans l'enfer de la contrainte, sous prétexte qu'elles nous empêcheraient d'être libres et nous maintiendraient sous leur emprise. Ce que l'on doit critiquer est non le devenir des institutions, mais certes leur caractère devenu , autrement dit quand elles n'offrent justement plus de devenir. Réflexe libertaire qui voit dans toute institutionnalisation un mal germer, un blocage dans l'invention du quotidien, un arrêt dans l'incessant renouveau du vivant. Nietzsche nous invite à contrôler pareil réflexe: conception erronée de ce que porte une institution - et en même temps si nécessaire, probablement si vitale. En renversant la croyance triviale qui institue que les institutions ne valent rien parce qu'elles ne seraient plus dignes de nous, c'est-àdire de notre liberté, Nietzsche nous rappelle comment nous avons en fait oublié la dette que nous avions envers les institutions. La valeur des institutions provient non seulement du pouvoir qui leur est dévolu, mais surtout de la dette qu'elles imposent aux individus qui les composent. Cette dette est le lien fondamental entre individu et institution. Une fois oubliée, il ne reste que le pouvoir par où l'institution bientôt nous échappe et nous oppresse. Plus exactement, ce ne sont pas les institutions qui imposent une dette: comment le 
pourraient-elles puisque leur pouvoir ne leur vient que de la dette d'abord allouée? Ce sont donc les individus qui, dans et par l'invention d'une institution, la constituent comme instance d'endettement. En inventant une institution, nous nous donnons une dette.

Prenons un exemple, l'exemple par excellence d'une origine des institutions en même temps que d'une institution de l'origine, et restons avec Nietzsche puisqu'il en donne un récit - qui est aussi une logique de la création des Dieux. La conviction s'est installée que c'est seulement par les sacrifices et les accomplissements de leurs ancêtres que la tribu existe et que l'on doit les payer de retour par des sacrifices et des accomplissements: on doit alors reconnaître une dette (Schuld) qui augmente constamment dans la mesure où les ancêtres ne cessent jamais, dans leur existence d'esprits puissants, d'accorder à la tribu de nouveaux avantages et de nouvelles forces (...). La peur des ancêtres et de leur pouvoir, la conscience d'être endetté envers eux augmente, selon cette logique, en proportion directe avec l'augmentation de pouvoir de la tribu elle-même (...). Si l'on imagine cette sorte de grossière logique poussée à bout, alors les ancêtres de la plus puissante des tribus sont contraints de prendre des dimensions monstrueuses dans l'imagination d'une peur croissante et de reculer dans la noirceur d'une irreprésentable et mystérieuse divinité : à la fin les ancêtres doivent nécessairement être transfigurés en dieux . (Généalogie de la morale, § 19)

Le mécanisme de l'institution des dieux recourt donc à cette double opération du pouvoir et de la dette. En allouant aux ancêtres un pouvoir qu'ils ne possédaient certes pas eux-mêmes, la tribu cherche à mettre à l'extérieur d'elle-même l'origine et la légitimation du pouvoir. Telle est bien la nécessité de la dette: elle permet de mettre hors de portée des membres de la tribu toute remise en cause de l'organisation sociale. En se donnant une dette, en s'inventant une dette envers une altérité de plus en plus radicale (ancêtres, esprits puissants, dieux enfin), on oublie que l'on était à l'origine de ce processus et du pouvoir. La conséquence est simple: on évite ainsi la tentation de produire un autre ordonnancement du social. La logique du processus est celle-ci: en se donnant une dette envers une altérité radicale que l'on a inventée, on s'ôte la possibilité de produire une quelconque altération du mode d'organisation sociale du sein même de la tribu.

Comme le remarque Wlad Godzich: "Nietzsche's account makes clear that the gods are the products of the society, 
and one can therefore speak of the social production of this otherness even though the form it takes is not a probut an ex-duction." [ $\underline{\mathbf{3}}$ ] Godzich poursuit en opposant la production collective des frontières entre altérité et société dans les sociétés à base religieuse, et la production sociale, non plus collective mais médiatisée par l'État, de ces mêmes frontières au sein des sociétés modernes. Là n'est pas mon propos et je préfère simplement insister sur cette très pertinente intuition qui fait revenir Godzich sur son énoncé: le fait que cette production collective prenne en fait la forme d'une exduction. Car par là aussi passe la différence entre société à base religieuse et société moderne: ici la légitimité repose sur une ex-duction, là sur une production; ici la tribu reçoit du dehors son ordre social, là la société produit de l'intérieur d'elle-même ce qu'elle doit être. Comme on l'imagine sans peine, cela engage deux relations fort différentes à la dette.

Dans une société régie par ex-duction, recevant du dehors sa loi, la dette opère à l'intérieur et de l'intérieur de la société : plus le pouvoir s'accroît, plus la dette grandit, mais aussi plus la dette est grande, plus le pouvoir augmente. Au contraire dans une société ordonnée par une pro-duction, inventant donc ce qu'elle est du dedans d'elle-même au lieu de le recevoir, la dette en vient à opérer à l'extérieur de la société, ou, plus précisément, elle en figure l'extériorité. Plus on oublie la dette, plus le pouvoir est grand.

Tel est ce qui fait, pour Nietzsche, que nous ne sommes plus dignes de nos institutions, que nous ne les valons plus: la dette envers les institutions a été oubliée et il ne nous est resté que la farce macabre du pouvoir, non plus la dette autorisante mais le pouvoir autoritaire. D'où aussi, soit dit en passant, une certaine fascination pour le modèle du pouvoir autoritaire, dans la mesure où il semble capable de faire l'économie de la dette, c'est-àdire de s'en passer. Cependant la dette ne cesse de resurgir aussitôt que le pouvoir autoritaire s'appuie sur une instance légitimatrice, que ce soit la patrie, la nation, la race ou l'histoire. Mais alors la dette n'est plus ce qui permettait une harmonie sociale en mettant à l'extérieur de la tribu, hors de portée du pouvoir immédiat des êtres, la légitimation du groupe et de sa puissance, la dette, dans ces circonstances, est ce qui engage l'altérité au sein même de la société. Nietzsche attirait l'attention sur le fait que, dans son schéma de l'origine des dieux, c'était en définitive la peur (et non la piété) qui avait conduit à leur invention: la peur du monstrueux dont on avait fait l'extériorité sociale par excellence. Désormais, dans les sociétés modernes, la 
peur de l'autre circule dans les relations sociales. Et cette peur, n'opérant plus en conjonction avec le sentiment de la dette, en est réduite à s'appuyer parfois sur la seule autorité du pouvoir et de la force. D'où les tentatives, dans les économies politiques modernes (encore qu'il n'y ait, à strictement parler, d'économie politique que moderne), pour penser un contrat social, afin de limiter la puissance des plus forts sur les plus faibles: on ne commence à saisir pareille nécessité qu'à partir du moment où la peur s'inscrit à l'intérieur des relations sociales, plutôt que de projeter sa crainte au dehors de la tribu.

Si l'on prend cette question de manière plus historique, et moins purement conceptuelle, on peut s'apercevoir que la valorisation sociale qui portait autrefois sur l'oisiveté et la dévalorisation implicite du travail (le travail n'était digne que des esclaves ou des personnes soumises aux nécessités de l'existence) se sont en quelque sorte retournées et croisées peu à peu, jusqu'à occuper des positions rigoureusement inverses: chez Locke, l'identité de l'homme ne tient plus seulement à sa raison, mais aussi à sa capacité de travail (animal laborans autant qu'homo rationalis), et, bientôt, chez Adam Smith, le travail occupe la place centrale de son économie politique. Le travail n'est pas même une valeur parmi d'autres, il est devenu la mesure de la valeur, la valeur qui rend équivalentes des valeurs jusque-là incommensurables. Si les valeurs respectives du travail et de l'oisiveté se sont ainsi lentement inversées, cela tient justement au déplacement dans la considération de la dette. Tant que l'on reconnaît, en effet, une instance extérieure à la société comme instance de légitimation de la communauté, comme l'origine de tout ce qui a été produit, de la matérialité des choses à celle des signes, la notion de production elle-même est située à l'extérieur de la tribu: tout ce que font les hommes consiste à recevoir, recevoir signes et choses. De là une valorisation essentielle de l'oisiveté. L'oisiveté n'est pas la paresse, mais l'état de réception, de disponibilité aux signes et aux productions divines.

Les études des anthropologues ont montré que les sociétés que l'on appelle sauvages et que l'on a longtemps conçu comme des sociétés de pénurie et de manque (justifiant de façon circulaire leur faible développement technique par leur quête immédiate de nourritures et leur quête immédiate de nourritures par l'absence de médiation technologique), ces sociétés sont en fait les véritables sociétés de loisir et assurément d'abondance. Abondance, non pour accumuler, mais uniquement pour manger à sa faim et dépenser 
grandement le reste dans les fêtes, autrement dit dans la reconnaissance de la dette. Acquérir plus que nécessaire est essentiel pour montrer sa puissance, dépenser plus qu'il ne faudrait est vital pour reconnaître la dette. Pierre Clastres avait prétendu que l'on avait là, non des sociétés sans État comme on l'avait jusqu'alors pensé, mais bien des sociétés contre l'État (c'est-à-dire refusant la division sociale qu'implique tout État) - Il ajoutait (ce qui a été moins remarqué): des sociétés non sans production, mais proprement contre la production.

Lorsque l'on va investir la légitimité sociale à l'intérieur de la société, plutôt qu'en son dehors, alors il devient nécessaire d'y fonder aussi la source de cette légitimité: ce sera l'homme, à la fois par sa raison et par son travail. Pareil travail ne cherche pas seulement à domestiquer la nature, à la transformer en objet de production, plus fondamentalement encore, c'est l'homme lui-même qu'il faut domestiquer, arraisonner, auquel il faut faire rendre raison (cette fois comme on dit aussi qu'il faut faire rendre gorge à quelqu'un): la dette est réinscrite à l'intérieur même de la raison et du travail de l'homme. Cependant cette dette est désormais ce qui nous oblige à produire, et avant toute chose, à nous produire nousmêmes sur la scène de la raison. D'où le nouvel intérêt que l'on porte à l'éducation. En particulier à partir de la fin du XVIIIe siècle, et en connexion avec l'idéalisme allemand, on le voit au principe selon lequel éduquer consiste avant tout à apprendre à apprendre , comme s'il s'agissait simplement de faire fructifier un capital rationnel toujours déjà présent, et refuser toute réception (que l'on juge forcément passive) de traditions héritées du passé. C'est en quoi on peut comprendre qu'il ait fallu attendre Leibniz pour énoncer ce principe des principes, le principe de raison, tout à fait contemporain de ce que Norbert Elias appelle la civilisation des moeurs, dont un paradigme est l'honnête homme à la fin du XVIIe siècle, autrement dit celui qui produit un travail sur soi, qui se modifie de façon à produire la juste réponse face à toutes les circonstances de la vie quotidienne, mais un travail transparent, un travail dont l'idéal est de disparaître de la vue, un dressage du corps et de l'âme si impeccable que tout semble naturellement donné. Semblable production de soi ne se fait cependant pas sans difficultés, ce dont témoignent le nouveau développement de l'intimité et de la sphère publique et leurs délicates relations.

Un autre témoignage se trouve dans la tentative de circonscrire le produit de la dette dans une discipline nouvelle - ou plutôt de donner à la production un lieu 
théorique, l'économie politique, et de thématiser la dette dans un autre espace qui, lui aussi, naît au même moment, l'esthétique[ $\underline{4}$ ]. Les deux disciplines ont bien un objet commun, toutes deux parlent de la valeur.

L'économie politique en traite à l'aune de la production et du travail, alors que l'esthétique, au moins jusqu'à Kant, est surtout une esthétique de la réception . On doit reconnaître pourtant que la notion de production ne va pas tarder à envahir le champ de l'esthétique. Or la pensée économique qui gravite autour de cette notion de production s'avère incapable de thématiser les questions du don, de la dette ou de la dépense, tout ce qui valorise l'oisiveté, sinon sous une forme paradoxale - et un paradoxe qui passe totalement inaperçu, celui de travail improductif!

Ainsi du texte fondateur de l'économie politique classique, The Wealth of Nations d'Adam Smith. Y sont distinguées deux sortes de travail, l'un productif, l'autre improductif: "The labour of the manufacturer $<$ productive labour > fixes and realizes itself in some particular subject or vendible commodity, which lasts for some time at least after that labour is past. It is, as it were, a certain quantity of labour stocked and stored up to be employed, if necessary, upon some other occasion. (...) The labour of the menial servant <unproductive labour>, on the contrary, does not fix or realize itself in any particular subject or vendible commodity. His services generally perish in the very instant of their performance, and seldom leave any trace or value behind them."[ $\underline{\mathbf{5}}]$ (II, 3, §1) Le travail productif est donc productif parce qu'il est capable de stocker, de mettre en réserve une trace de temps, le temps du travail luimême, imprimé sur l'objet, et qui le transfigure en marchandise: la valeur vient de ce que le temps s'y trouve bloqué, mis de côté, arrêté. À l'inverse le travail improductif est une pure dépense de temps, une consommation immédiate du temps, la consommation instantanée de l'instant du travail, du maintenant. La dépense n'est pensable que négativement, comme ce que n'est pas le travail productif, ce travail qui opère comme modèle fondamental, ce qui assure justement la richesse des nations. Le code naissant de l'économie politique apparaît déjà si puissant, en son articulation avec le concept de production, qu'on n'aperçoit même pas le paradoxe d'un travail improductif, d'un travail qui ne fait rien, sous prétexte qu'il ne laisse pas forcément de traces matérielles derrière lui.

Ce caractère idéologique se perçoit encore mieux dans une autre opposition qui vient redoubler celle des deux 
formes de travail, celle de l'épargne et de la prodigalité, ou encore de la frugalité et de l'oisiveté: "Parsimony, and not industry, is the immediate cause of the increase of capital (...). Whatever industry might acquire, if parsimony did not save and store up, the capital would never be greater. By what a frugal man saves, he not only affords maintenance to an additional number of productive hands, (...) he establishes as it were a perpetual fund for the maintenance of an equal number in all times to come. (...) The prodigal perverts it in this manner. By not confining his expence within his income, he encroaches upon his capital. Like him who perverts the revenues of some pious foundation to profane purposes, he pays the wages of idleness with those funds which the frugality of his forefathers had, as it were, conservated to the maintenance of industry." (II, 3, 16-19). L'épargne est donc, à l'instar du travail productif, un stockage de temps, une trace de temps inscrite sur le corps des choses et que l'on garde pieusement, c'est la conservation d'un fragment de temps qui est susceptible de se conserver lui-même dans le temps et d'assurer ainsi la conservation de l'espèce. Le propos de Smith n'est pas trivialement économique, il a pour ambition de déterminer les conditions d'un vivre-ensemble harmonieux dans la suite des temps. On ne peut alors que remarquer combien le vocabulaire religieux vient imprégner tout ce discours. Ainsi le terme de "perpetual fund" désigne généralement les fonds laissés par testament pour des messes chantées pour le repos des âmes, mais surtout c'est ici avec la qualification de la dépense comme perversion de la frugalité des ancêtres, de cette pieuse fondation dilapidée dans l'oisiveté, qu'on a un retournement total du lexique et des valorisations des sociétés à base religieuse: la pieuse fondation ne recouvre plus la dette nécessaire envers les dieux ou les ancêtres, la dépense et l'oisiveté dans lesquelles il fallait se maintenir, elle est désormais fonction de l'épargne et de la conservation du temps.

Il reste bien une dette envers les ancêtres, cependant loin de conduire à dépenser pour eux, par respect pour eux et en leur honneur, mais aussi par crainte de leur puissance, il s'agit au contraire de produire et d'accumuler. Non plus donner des présents aux instances sacrées du passé, mais produire du présent et le faire ainsi passer dans l'ordre des signes et des marchandises, conserver le présent dans le futur. C'est pourquoi Adam Smith part en guerre contre la dilapidation du présent: "With regard to profusion, the principle which prompts to expence, is the pression for present enjoyment, which though sometimes violent and very difficult to be restrained, is in general only 
momentary and occasional. But the principle which prompts to save, is the desire of bettering our condition, a desire which, though generally calm and dispassionate, comes with us from the womb, and never leaves us till we go into the grave" (II, 3, 27). A la dépense s'oppose donc l'épargne, comme au divertissement et à l'enjouement, à la jouissance du moment présent s'oppose le désir d'améliorer sa condition, ou encore comme la passion violente et difficile à contrôler au désir calme et dépassionné, un désir éminemment raisonnable. Mais le plus remarquable tient au redoublement des oppositions temporelles où l'intérêt pour un certain mode temporel est dupliqué par la temporalité spécifique de cet intérêt: la passion pour le moment présent ne saurait être elle-même que momentanée et occasionnelle, tandis que le désir d'améliorer sa condition, et donc de stocker du temps, relève lui-même d'une continuité temporelle qui entraîne tout individu de sa naissance à sa mort. Or c'est justement ce redoublement qui va permettre de valoriser le désir d'épargner par rapport à la passion de dépenser (outre bien sûr les connotations évidentes des couples passion/sans passion, violent/calme), dans la mesure où il est rattaché à un argument statistique: "though the principle of expence, therefore, prevails in almost all men upon some occasions, and in some men upon almost all occasions, yet in the greater part of men, taking the whole course of their life at an average, the principle of frugality seems not only to predominate, but to predominate very greatly." (II, 3, 27)

L'épargne, impliquant un exercice de continuité et de durée, s'avère donc dominer la pure intensité passionnelle de l'instant qui ne peut offrir que sa discontinuité et sa fragmentation. L'épargne, en tant que conservation du temps et de l'espèce, apparaît comme un principe de vie, alors que la passion de l'instant ne joue qu'à l'instar d'un principe de perte de soi et, au bout du compte, de mort. Ce qui condamne l'instant, la pure occasion, c'est qu'ils n'ont pas d'avenir. Aussitôt éclos, ils disparaissent sans laisser de trace, aspirés qu'ils sont par la violence du présent. Non seulement ils impliquent une absence d'avenir, mais encore une perte du passé, puisque les fonds perpétuels des ancêtres s'y évanouissent aussi vite. A l'inverse, l'épargne conserve le passé et se conserve dans l'avenir. Plus encore, elle est une ouverture de l'avenir, dans la mesure où elle permet une amélioration générale des conditions.

Pour nous, il est devenu évident que production et épargne sont les valeurs à l'aune desquelles nous mesurons tout le reste. Mais une semblable 
condamnation de la dépense et de l'oisiveté, même préparée par toute une série d'inflexions historiques, n'en demeure pas moins frappante et contraire à que nous savons des sociétés antiques ou des sociétés traditionnelles en général. Ainsi le grec ancien ne possède aucun terme correspondant à notre travail (au sens d'activité productrice). Ponos désigne en fait toutes les activités réclamant un effort, et pas seulement le labeur du travailleur. L'ergon peut se rapporter aux travaux des champs (dans l'expression au pluriel ta erga), mais il renvoie toujours au fait, pour chaque chose, d'être le produit de sa vertu propre, le développement de son arete. Quant au poiein, il désigne surtout la fabrication artisanale, technique, en s'opposant au prattein, qui ne consiste jamais à produire quelque chose, mais uniquement à dérouler une activité pour elle-même, en être l'usager et non le producteur. C'est pourquoi Vernant souligne que l'idéal de l'homme libre, de l'homme actif <celui qui est dans la praxis>, est d'être universellement usager, jamais producteur. Et le vrai problème de l'action, au moins pour les rapports de l'homme avec la nature, est celui du "bon usage" des choses, non de leur transformation par le travail[ $\underline{\mathbf{6}}$ ] . Cela au moins pour une raison, c'est qu'il n'est pas question, pour un Grec, de considérer l'être humain comme pouvant posséder le temps, il n'en saurait être que l'usager. Or, pour Smith, si le temps est épargnable, stockable, par le travail humain, c'est justement que l'homme peut avoir prise sur lui. C'est en ce sens que l'oisiveté devient condamnable puisqu'elle repose sur le principe d'une immaîtrise du temps: tout son art réside dans le fait de savoir être dans le temps, certes pas d'enfermer du temps dans des objets.

Pour les Grecs, l'oisiveté est valorisée parce qu'elle seule permet de demeurer attentif au temps, et d'en user ainsi à bon escient, d'en saisir l'occasion (quoique saisir soit déjà trop dire, supposant une maîtrise, une mainmise: plutôt laisser être l'occasion et se laisser être soi-même dans l'occasion, l'instant, le bon moment, le juste présent). Même pour l'artisan, oeuvrant plus dans le prattein que dans le poiein, le temps de l'opération technique n'est pas une réalité stable, unifiée, homogène, sur quoi la connaissance aurait prise; c'est un temps agi, le temps de l'opportunité à saisir, du kairos, ce point où l'action humaine vient rencontrer un processus naturel qui se développe au rythme de sa durée propre . (Vernant, p. 52). Platon insiste sur la nécessité, pour l'artisan, de ne pas quitter sa tâche, sous peine justement de rater le bon moment, l'opportunité du kairos. Cela dit le fabricant qu'est l'artisan demeure l'esclave du kairos, précisément en ce qu'il reste dans 
l'ordre de la production d'objets. Alors que le sophiste, dont l'enseignement concerne la praxis se donne au contraire comme le maître du kairos, ou plutôt, comme celui qui sait s'ajuster parfaitement au rythme propre du kairos, celui qui sait le reconnaître et en user. Il ne domine pas le kairos, il en figure le parfait usager. Telle est donc la valeur de l'oisiveté: se tenir prêt au juste usage du kairos. A l'ordre de valeurs que constituent la contemplation, la vie libérale et oisive, le domaine du naturel, la culture grecque oppose, comme autant de termes négatifs, les catégories dépréciées du pratique, de l'utilitaire, du travail servile et de l'artificiel. (ibid., 43)

C'est ce que l'on voit exemplairement chez Aristote lorsqu'il valorise, dans l'Éthique à Nicomaque, le magnifique aux dépens non seulement de l'homme libéral (prêt à donner et à dépenser), mais surtout du parcimonieux (qui est aussi bien l'avare, celui qui refuse de donner, que le pillard ou le joueur, qui n'ont en vue que l'appât du gain). Il ne s'agit pourtant pas d'être prodigue n'importe comment et envers n'importe qui. Le Magnifique est certes celui qui est capable de donner énormément, il est surtout celui qui donne à bon escient : c'est un artiste en fait de dépense, celui qui reconnaît la dette de la société en son entier (qui la reconnaît au double sens de connaissance et d'acquittement). Le Magnifique s'avère supérieur à l'homme libéral en ce qu'il a les moyens financiers de donner beaucoup plus que celui-ci, mais plus encore parce que cela lui permet de rendre les dettes de la communauté envers les dieux et les ancêtres: Le magnifique en effet ne dépense pas pour lui-même, mais dans l'intérêt commun, et ses dons présentent quelque ressemblance avec les offrandes votives . (Éthique à Nicomaque, IV, 5, 1123 a 5). Et, dans la dernière partie, lorsque Aristote s'interroge sur le meilleur état pour l'homme, c'est sans conteste la vie contemplative, c'està-dire une existence de loisir qu'il met au sommet des conditions sociales: Cette activité paraîtra la seule à être aimée pour elle-même: elle ne produit (ginetai), en effet, rien en dehors de l'acte même de contempler (theoresai), alors que des activités pratiques nous retirons un avantage plus ou moins considérable à part de l'action elle-même. De plus le bonheur semble bien consister dans le loisir (skhole): car nous ne nous adonnons à une vie active qu'en vue d'atteindre le loisir, et ne faisons la guerre qu'afin de vivre en paix . (Éthique à Nicomaque, X, 7, 1177 b 1-5). Le loisir n'est pas important seulement parce qu'il autorise une disponibilité aux signes du monde et une attention à la venue du kairos, il n'opère pas uniquement sur le mode 
d'un cadre temporel et social, il permet d'appréhender la juste manière de régler ponctuellement ses dettes envers ce qu'envoient les dieux, de saisir l'instant de leur don comme une dette, de réaliser que le loisir s'offre luimême comme une dépense de temps, un refus de faire produire le temps. Le loisir est à la fois condition de possibilité de la prise en compte de la dette et reconnaissance implicite de la dette par la dépense de temps qu'il implique. Etre dans le loisir, c'est entrer déjà dans le temps de la dette. Et admettre aussi que cette dette n'est jamais remboursable.

$\mathrm{Au}$ moment où l'on pense que le temps est maîtrisable, appropriable, la relation à la dette change considérablement. Durant le Moyen Age, le travail reste certes dévalorisé par rapport au loisir de la vie contemplative, monastique et l'usure est systématiquement condamnée en ce qu'elle implique une appropriation illégitime du temps qui n'appartient qu'à Dieu. Pourtant, peu à peu, travail, production, profit usuraire s'imposent comme des activités de valeur sous la force du développement agricole, des échanges marchands et d'une structure féodale qui déplace le statut des travailleurs et du servage, sous la force aussi de la religion chrétienne qui, en remodelant les rapports entre ciel et terre à partir de la réforme grégorienne provoque un nouvel intérêt spirituel pour les choses terrestres. C'est ainsi que, si l'on a connu depuis longtemps des critiques du mauvais loisir (visant non la dépense, mais la dissipation du temps : dans la mendicité par exemple), on trouve dans certains textes de mystiques du XIVe siècle toute une condamnation, en termes typiquement mercantiles, de la perte de temps dans le loisir, et la mise en place d'une comptabilité spirituelle du temps (chez le dominicain pisan Domenico Cavalca, par exemple, dans sa Disciplina degli Spirituali).

Dès l'aube de la Renaissance, le temps a changé de mains pour ainsi dire: il n'est plus la possession de Dieu, mais celle de l'homme. Comme l'affirme Alberti dans son Libri della Famiglia, il y a trois choses que l'homme peut dire lui appartenir en propre: la fortune, le corps et le temps[ $\underline{7}]$. Par ailleurs l'horloge, qui prend de plus en plus de place à partir du XIVe siècle, est devenu l'emblème par excellence de l'humaniste: celui qui sait régler son temps. Le renversement est tel qu'au XVIIIe siècle, au moment de penser Dieu, on le concevra justement sous la forme de cette figure éminente du contrôle humain sur le temps: Dieu devient un grand horloger, le champion de la machinerie du temps. Cela ne signifie pas pourtant que le loisir disparaisse 
rapidement du réseau des valeurs sociales, mais de réaménagement en déplacement, il perd peu à peu sa position suprême. Il faut désormais l'établir sous forme de loi: ainsi la dérogeance apparaît-elle à la fin du Moyen Age comme un moyen de maintenir un statut social du loisir. Ou encore, chez les commerçants italiens s'instaure l'habitude de diviser en deux l'emploi du temps: le matin, les affaires, l'après-midi, les loisirs emploi qui persiste dans les relations mondaines: c'est ainsi qu'on ne saurait, sauf à être son amant, visiter une dame le matin. Ce n'est guère avant le XVIIIe siècle que le loisir occupe une position franchement dévalorisée et que travail et production prennent sa place sur le devant de la scène sociale. Il est significatif que Rousseau, dans une note de bas de page de son Essai sur l'origine des langues, récupère le paradoxe aristotélicien de la guerre poursuivie pour mieux vivre en paix, comme la vie active pour mieux accéder au loisir, avec une modification qui en déplace radicalement la portée: Il est inconcevable à quel point l'homme est naturellement paresseux. On dirait qu'il ne vit que pour dormir, végéter, rester immobile; à peine peut-il se résoudre à se donner les mouvements nécessaires pour s'empêcher de mourir de faim. Rien ne maintient tant les sauvages dans l'amour de leur état que cette délicieuse indolence. Les passions qui rendent l'homme inquiet, prévoyant, actif, ne naissent que dans la société. Ne rien faire est la première et la plus forte passion de l'homme après celle de se conserver. Si l'on y regardait bien, l'on verrait que, même parmi nous, c'est pour parvenir au repos que chacun travaille; c'est encore la paresse qui nous rend laborieux[ $\underline{\mathbf{8}}$ ] . Le repos de la contemplation s'est muée en paresse de l'existence improductive. C'est qu'il s'agit désormais, avant tout, de ne plus perdre son temps en le soumettant à des calculs rationnels s'exprimant en valeur-travail. Tel est le nouvel équivalent général des pratiques humaines, ce qui autorise et légitime le code de l'économie politique[ $\underline{\mathbf{9}}$ ].

Ce bouleversement du rapport au temps déplace aussi la relation à la dette. Dans l'ancien rapport aux dieux ou aux ancêtres, on était dans le temps - et même si ce temps n'était pas le même que l'âge d'or des dieux, il y trouvait son origine. Sans doute la distance des dieux aux hommes restait grande, mais le temps des hommes se fondait dans celui des dieux dans la mesure où c'étaient les dieux qui donnaient le temps (ce qui leur allouait aussi la possibilité de s'y introduire au besoin ici ou là, demain ou hier). Avec ce réaménagement du temps et la désimbrication du ciel et de la terre, des dieux et des hommes, le temps devient une possession humaine, mais la dette ne peut plus être soumise au 
même poids du passé. La pesanteur du passé qui, dans une société traditionnelle, permet de maintenir les hommes dans un champ homogène, apparaît en fait de plus en plus pesante.

Le passé n'est plus ce qui fonde l'unité des personnes, ce qui les rassemble dans une communauté d'expériences et d'apprentissages, il est maintenant vécu comme un obstacle au sain développement de l'individu et de la société. On ne reconnaît plus une dette envers le passé, on cherche à investir dans l'avenir. Il devient dès lors essentiel, dans ce mouvement qui prend la figure récente $\mathrm{du}$ processus et qui s'appuie avant tout sur une dynamique du nouveau, d'oublier la dette envers le passé [ 10 ]. La dette se trouvera alors déplacée et prise en charge par une nouvelle discipline, l'esthétique, dans la mesure où il s'agit de thématiser la question de la valeur en la maintenant dans l'orbe du loisir; cependant il est aussi un autre lieu discursif, un autre territoire disciplinaire, portant un nom vénérable, mais dont les procédures et les opérations de légitimation vont subir une mutation d'importance au cours des XVIIIe-XIXe siècles, et qui va servir à reconfigurer la dette envers le passé: l'histoire.

Avant d'y venir explicitement, un dernier passage de Nietzsche, intitulé Loisirs et oisiveté , extrait cette fois du Gai savoir : "Mieux vaut agir que ne rien faire", voilà encore un de ces principes chargés à balle qui risquent de porter le coup de grâce à toute culture supérieure, à toute suprématie du goût. Cette frénésie de travail sonne le glas de toute forme; pis elle enterre le sentiment même de cette forme, le sens mélodique du mouvement. (...) Car la vie, devenue chasse au gain, oblige l'esprit à s'épuiser sans trêve au jeu de dissimuler, de duper ou de prévenir l'adversaire; la véritable vertu consiste maintenant à faire une chose plus vite qu'un autre. (...) Chaque jour le travail accapare davantage la bonne conscience à son profit. (...) Eh bien, autrefois, c'était le contraire: c'était le travail qui donnait des remords. Un homme bien né cachait le sien, si la misère le contraignait à en faire un. L'esclave travaillait, accablé par le sentiment de faire quelque chose de méprisable: "faire" à lui seul l'était déjà. "Il n'est de noblesse et d'honneur que dans le loisir et la guerre": ainsi parlait le préjugé des anciens . (§ 627) Tout en laissant de côté le préjugé nietzschéen selon lequel le travail s'opposerait au goût de la culture supérieure, alors même que goût et culture sont bien les produits d'un identique mouvement historique et résultent du travail plus qu'ils ne s'y heurtent, ce qui m'intéresse ici, outre la très juste 
révolte de Nietzsche contre l'arrogance du travail et l'oubli de l'oisiveté, c'est la référence au faire, au sens d'une fabrication et d'une production, dans la mesure où elle permet d'entendre le scepticisme nietzschéen à l'égard de l'histoire.

L'histoire - pour y venir maintenant - est en effet liée à l'idée d'un faire. Si l'historiographie moderne se détache des modèles anciens et finit par s'institutionnaliser à la fin du XVIIIe siècle, ses premiers mouvements sont en fait contemporains de ce déplacement qui rend l'homme producteur de sa propre histoire par son travail et sa raison. Faire de l'histoire l'historiographie - n'est pas indépendant, en son principe, de la façon dont les hommes s'avèrent désormais susceptibles de faire l'histoire. Les événements historiques ne sont plus reçus et rapportés afin de servir d'exemples de conduite, faire l'histoire implique la prégnance d'une volonté que l'historien, lorsqu'il veut en faire l'histoire, doit s'attacher à suivre, à mettre en scène et à comprendre. Mais si l'agir historique requiert une volonté, il réclame aussi un savoir de la société sur laquelle exercer son action, il a besoin de modèles d'intelligibilité du social. Réciproquement l'intelligibilité historique est l'exemple même d'une volonté de savoir .

Le savoir occupe en effet une position nouvelle à partir de la Renaissance, une position de plus en plus centrale qui permet de maintenir une unité de la vieille Europe que la chrétienté avait assurée durant plusieurs siècles et qu'elle ne parvient plus à légitimer. En même temps le savoir devient objet de conquête et témoigne de maintes luttes pour le contrôler, dans la mesure où, d'une part il est associé de près au développement de l'État moderne, d'autre part il permet d'établir des différences sociales. D'où, tout au long du XVIIe siècle, une constante querelle entre les savants, héritiers des modèles scolastiques, et les mondains qui prétendent au savoir de l'homme social. Au cours du XVIIIe siècle, l'histoire en vient à jouer un rôle de plus en plus important au sein de ce savoir, car elle permet d'entériner et de rendre compte du mouvement constitutif des temps modernes, puis de la modernité, à savoir la dissociation entre passé et présent. L'âge moderne, formé à l'invention de soi, ne saurait s'accommoder des modelages anciens: il faut que chaque présent affirme sa puissance de fondement, satisfasse sa soif créative sans puiser à la source du passé. S'il est une différence majeure dans l'usage de l'historiographie, elle réside là: pour les Anciens, l'histoire - quelque exacte et véridique qu'elle se veuille a pour fonction éminente d'enseigner, il faut qu'elle 
s'établisse comme un bien pour toujours ainsi que le souhaite Thucydide, et Cicéron le reformule exemplairement en faisant de l'histoire la magistra vitae, l'éducatrice de la vie. L'historiographie est avant tout un répertoire d'exemples à suivre, le cens et le sens de modèles d'expérience, puisque le sens des expériences repose sur leur possible recensement, et leur cens est déterminé par le sens alloué à ce qui est reconnu comme modèle d'expérience. On ne fait pas (de) l'histoire, on en reçoit les expériences. Cette réception est affaire d'autorité. Et il faut bien reconnaître que les historiens médiévaux ont parfois bien du mal à juxtaposer ou entrelacer les enseignements des Saintes Écritures et des auteurs païens de l'Antiquité. Mais peu importent les contorsions conceptuelles, on ne peut comprendre ces curieuses façons si l'on ignore qu'elles répondent à la nécessité de ne pas dissocier l'enseignement du passé des pratiques du présent.

Or à partir du XVIe siècle, c'est le statut même de l'exemplarité qui change, de conserve avec le nouvel investissement dans la subjectivité. De la même façon que, chez Cicéron, l'historiographie fournit un stock d'exemples pour l'orateur ( Plena exemplorum est historia ), au Moyen Age s'est développée, surtout aux fins de prédication, la composition de recueil d'exempla, petits récits donnant de façon exemplaire un cas historique passant pour modèle d'existence. Cette valeur des exempla se perd peu à peu jusqu'à devenir opaque au XVIIIe siècle: la vie d'un homme n'étant plus réglée sur des modèles à pratiquer, on ne sait plus s'arrêter dans le fourmillement d'événements qui forment l'existence d'un individu et en marquer les inflexions majeures, les moments exemplaires. A la différence des hagiographies médiévales, les biographies au XVIIIe ne peuvent plus nous apprendre d'une vie que le mouvement incessant de la vie ( exemplaire de ce point de vue est la Vie de Samuel Jonhson de Boswell). De l'exemplum, on est passé à l'anecdote, ce petit détail insignifiant que l'on espère désespérément significatif et, bien sûr, comme Boswell, on tente de renverser l'aporie en affirmant que, d'un homme de génie, tout devient révélateur. Ce qui impose tout un appareillage de modes de lecture, toute une herméneutique de l'anodin.

Que j'illustre cela par une anecdote: Lors d'une conférence qui se tenait à Charlottenburg, Oelssen <chef du département au Ministère des Finances> prit vivement parti pour la fabrication massive de papiermonnaie afin d'éponger les dettes. Voyant que tous les arguments contraires ne faisaient aucun effet, j'avançai 
(connaissant bien mon homme) avec une belle hardiesse l'argument suivant: "Mais Monsieur le Conseiller d'État secret, vous vous souvenez certainement que Thucydide raconte déjà quels maux sont nés du fait qu'Athènes avait fabriqué trop de papier-monnaie" - "Voilà une expérience d'une grande importance" me répondit-il en me donnant raison[ $\mathbf{1 1}$ ]. On voit combien l'exemplarité de la référence historique opère encore de façon déterminante sur le Conseiller d'État, mais on perçoit aussi toute la distance du savant historiographe qui est capable de jouer avec ce qui apparaît désormais comme un inutile préjugé, bon seulement pour les ignorants et les présomptueux. C'est parce qu'il connaissait son homme , manifestement respectueux de l'érudition historique et de son enseignement, mais pas assez connaisseur de l'histoire, que le coup rhétorique marche. Quant à l'ancien respect de la dette envers le passé, il ne sert plus qu'à éviter l'alourdissement de la dette économique présente.

L'histoire a bien pour but de rendre le passé, mais la dette qui s'y trouve impliquée, semble de plus en plus oubliée. Car le processus historiographique se fonde sur une double altérité temporelle ou une double altération du temps. Pour que l'histoire, telle que nous la connaissons encore, apparaisse, il faut d'abord rendre le passé étranger au présent, instaurer donc une coupure radicale entre les deux temps, rendant ainsi nécessaire un regard rétrospectif, et, non plus, comme dans la tradition, une actualisation constante du passé qui fait passer l'anachronisme au rang de principe, catapultant le passé dans le présent. Pour le dire vite: la tradition maintient le passé vivant dans le présent en en altérant la marque propre, l'historiographie est l'écriture présente d'un passé mort, voire de la mort du passé intact. La tradition, c'est le baiser de la princesse qui transforme le crapaud du passé dans le prince charmant du présent; l'histoire, c'est la Belle-au-Bois-dormant qui attend, intouchée dans le lit du temps, le baiser de l'historien pour se réveiller, mais on sait que, dans ce conte de fées, la mère de l'historien-prince charmant n'est autre qu'une ogresse qui rêve de dévorer la chair merveilleusement fraîche du passé.

Comme le dit Michel de Certeau, l'histoire moderne occidentale commence en effet avec la différence entre le présent et le passé. Par là, elle se distingue de la tradition (religieuse), dont elle ne parvient jamais à se séparer tout à fait, entretenant avec cette archéologie une relation d'endettement et de rejet[ $\underline{12}$ ] . En effet si l'histoire veut rendre le passé, elle est endettée envers la façon dont la tradition parvenait à maintenir vivant le 
passé par la reconnaissance immédiate et implicite de ses dettes envers son passé - mais elle ne peut qu'en rejeter et oublier l'efficace, dans la mesure où il n'est plus question, pour elle, de reconnaître cette dette envers des ancêtres ou des dieux, de chercher un exemple ou un modèle de vie dans les histoires anciennes, d'endosser l'intelligence du passé. Ce n'est plus le passé qui rend intelligible le présent, c'est le présent qui invente des modèles d'intelligibilité du passé. L'histoire n'admet sa dette que dans son désir de rendre vivant, de rendre présent, un passé qu'elle s'est appliquée auparavant à enterrer. La manière d'oublier et de reconnaître sa dette est donc identique, elle se fonde sur les procédures d'enquête de l'historiographie, en même temps que sur sa capacité à ordonner les résultats de la recherche de manière à faire apparaître du sens.

Car en plus de cette première altérité temporelle, rendre irréductiblement étrangers passé et présent, il est nécessaire d'en produire une seconde qui légitime le regard historiographique: rendre le passé étranger à luimême. L'histoire moderne suppose en effet que le passé ne se connaît pas lui-même: le temps de la production n'est pas le temps du savoir. Chaque présent s'avère en fait aveugle à ce qu'il est, dissipé aussitôt dans l'immédiateté, incapable de se soulever hors de son fugitif présent pour apercevoir le paysage qu'il compose, puisque lui ferait défaut le levier historique de la médiation: il n'est de connaissance que d'autrefois, jamais d'aujourd'hui. Rendre le passé étranger à luimême est essentiel pour prétendre pouvoir rendre le passé (tout court). Cela implique, bien sûr, que, du présent, on ne peut avoir de connaissance distincte et panoramique, seulement des impressions diffuses et des esquisses locales. C'est là ce qui donne à l'historien son pouvoir souverain, son coup d'oeil d'expert sur le passé: seul, il peut rendre le passé, dans la mesure où il est à même de le pro-duire sur la scène du présent. Si c'est là la conception moderne de l'histoire, cela ne signifie pas que, dans les sociétés traditionnelles comme la société médiévale, on croyait à une connaissance immédiate du présent. D'une part parce qu'il n'y avait pas l'investissement moderne sur le savoir comme légitimation des pratiques quotidiennes et institutionnelles; d'autre part, parce que, si l'on concevait bien une différence entre production et réception de signes, et si les personnes se situaient plus du côté de la réception, Dieu assurait au moins qu'à la fin des temps l'homogénéité, l'adhérence de la production et de la réception aurait lieu. Le présent n'avait donc pas à rendre le passé, mais à reconnaître sa dette envers lui par son actualisation même. $\mathrm{Au}$ 
contraire l'histoire occidentale moderne est contrainte de rendre le passé, faute de pouvoir gérer autrement sa dette que sous la forme d'un oubli et d'une restitution. Le passé opère comme une figure de l'extériorité du présent. Il faut alors, pour l'historien présent, travailler sur le passé de manière à nous le rendre à la fois étranger et proche. Le travail historiographique consiste à nous faire savoir que, sous les mêmes mots, sous les mêmes figures, sous les mêmes institutions, se cachent au fil du temps des différences radicales, des altérités monumentales, des incompréhensions totales.

L'historien a pour tâche de nous révéler combien le passé est étrange, combien il n'est plus - et pourtant, il veut nous amener à sa proximité, comme pour une visite, sous les apprêts endimanchés du savoir, au cimetière de l'autrefois. Il prétend nous faire faire l'expérience de l'autre.

Cette expérience procure à la fois apaisement et inquiétude, sécurité et torture. On le discerne sans peine dans les citations de Michelet que donne de Certeau:

l'intimité avec l'autre monde est désormais sans danger dans la mesure où la dette envers les ancêtres est oubliée, dans la mesure où la peur qui était à l'origine des dieux s'est perdue. Mais en même temps ce silence des ancêtres voue les survivants historiens à la mélancolie de voix à jamais effacées de leur puissance, de voix que l'on ne peut jamais vraiment entendre.

Comme le résume de Certeau: l'historiographie suppose qu'il est devenu impossible de croire en cette présence des morts qui a organisé (ou organise) l'expérience de civilisations entières, et qu'il est pourtant impossible de "s'en remettre", d'accepter la perte d'une vivante solidarité avec les disparus, d'entériner une limite irréductible (p. 12). Là où il y avait dette et présence des ancêtres, la dette est désormais perdue comme telle et les anciens sont absents. On pourrait croire que tout va pour le mieux dans le meilleur des mondes possibles, puisque l'on s'est débarrassé d'une encombrante dette, inutile au fond, puisque l'origine de toute production, y compris celle des dieux, vient de nous. Cela permettrait alors d'éviter cette curieuse contorsion mentale qui consiste à se donner soi-même une dette envers un autre afin de mieux s'obliger et se contraindre. Et pourtant le gain à se décharger de la dette n'a rien d'évident, puisque, du coup, nous voilà hantés par le deuil du passé. Ce travail de deuil auquel nous sommes astreints, nous le nommons histoire . Car il s'agit bien d'un travail, d'un lieu de production. Rendre le passé n'est pas le redonner, encore moins le donner, c'est le produire par la force de travail de l'écriture. Le savoir en est la plus-value. 
L'écriture substitue aux représentations traditionnelles qui autorisaient le présent un travail représentatif qui articule en un même espace l'absence et la production (ibid.) Autrement dit, le présent n'est plus autorisé par le passé de la tradition, ce qui donnait à chaque présent l'impression de participer aux fêtes du passé parce qu'il participait justement de l'inaltérable présence du passé. Impression en un double sens: et le passé marquait de son sceau la chair du présent en un rituel au besoin cruel (la mémoire fonctionne à la douleur), et la participation du présent au passé opérait, non selon une pure répétition, mais selon une actualisation où l'invention du nouveau ne pouvait se faire que sous la guise de l'autorité ancienne, donnant donc seulement l'impression que le passé se répétait. Or c'est cette manière de situer le présent dans la continuité du passé, afin d'en reprendre (ou d'en usurper) l'autorité, qui se décompose lentement et se trouve remplacée par la revendication d'une discontinuité des temps. Et si ce travail, nommé histoire , parvient à articuler absence et production, c'est d'abord parce que l'écriture historique produit le passé comme absence et se produit elle-même dans l'absence du passé.

Existerait alors une autre différence entre tradition et histoire: celle-ci réclame un espace où articuler absence et production, passé et présent, alors que celle-là implique une temporalité où s'amalgament et s'enchevêtrent présence et tradition, passé et présent. L'histoire travaille pour beaucoup à partir de catégories spatiales, d'où son lien depuis longtemps effectif avec la géographie, d'où aussi sa relation au pouvoir et à la mise en place des États modernes, dans la mesure où ceux-ci asseyent leur légitimité et leur autorité sur une mesure de l'espace: la nation que contrôle et produit l'État est avant tout un territoire. Même l'idée de distance, qu'elle soit critique ou temporelle, inscrit en fait les rapports de pouvoir et de savoir dans l'espace: il n'est de distance, authentiquement, que spatiale. Le passé ne devient historique qu'à partir du moment où l'on pense, où l'on s'assure que, très littéralement, il a eu lieu. Pour autant la tradition ne se dissocie pas de la notion de lieu , bien au contraire: le passé lui apparaît avant tout comme un répertoire et une pragmatique des lieux. La tradition repose sur une topique, c'est-à-dire sur un art des topoï, des lieux communs. Mais, avec les temps modernes, ce sont justement ces lieux communs, cette communauté des lieux qui est questionnée et soupçonnée, avec laquelle on prend ses distances. Le lieu commun, riche d'expérience accumulée, se change 
en cliché, reproduction vide de sens là où on attend une production du nouveau - ou une production du passé, mais sous la forme d'une nouvelle vision du passé, d'un nouveau sens de l'histoire, autre manière de prendre ses distances avec le passé tout en le ressuscitant, ou parce qu'on le ressuscite. Dans la tradition, la topique est une pragmatique du quotidien; pour l'histoire, elle devient la forme rhétorique de son inscription. Pour que l'écriture de l'histoire ait lieu, occupe un lieu institutionnel, il faut mettre à distance le passé et par là-même prendre ses distances vis-à-vis de la dette.

La dédicace, plus ou moins discrète (il faut maintenir la fiction du passé pour que le jeu savant de l'histoire ait "lieu"), affecte au discours son statut d'être endetté par rapport au pouvoir qui, hier était celui du prince, et aujourd'hui, par délégation, celui de l'institution scientifique d'État ou de son éponyme, le patron. Cet "envoi" désigne la place autorisante, le référent d'une force organisée, à l'intérieur et en fonction de laquelle l'analyse a place. Mais le récit lui-même, corps de la fiction, marque aussi, par les méthodes employées et par le contenu traité, d'une part une distance par rapport à cette dette, et d'autre part les deux points d'appui qui permettent cet écart: un travail technique et un intérêt public (id., p. 18). La dette envers l'institution prend donc la forme d'une reconnaissance d'un pouvoir autoritaire plutôt que celle d'une dette autorisante, et impose à l'historien, non une participation à cette dette, mais une tentative de s'en distancier, de travailler l'institution par son propre travail. Cependant si de Certeau reconnaît parfaitement cette logique de la dette, de l'institution, du travail, de la production et de la mort qui ordonne la figure et la pratique de l'historien, il entend trouver dans la valeur-travail, dans le travail productif de quoi penser l'histoire, le discours de l'histoire, mais aussi sa valeur propre, son intérêt intrinsèque. Ce qui le force brusquement à coller au texte, historiquement déterminé, de l'économie classique (Marx ici, mais Smith avant lui) en validant la référence au travail productif et en évoquant la possibilité d'y penser le discours dont il avoue, qu'à strictement parler le langage de l'économie politique, on ne devrait l'exprimer qu'en termes de travail improductif : Il m'a semblé qu'il était possible de transposer ici ce que Marx appelle le "travail productif" (...). Que le discours entre plutôt, chez Marx, dans la catégorie de ce que génère le "travail improductif" n'empêche pas d'envisager la possibilité de traiter en ces termes les questions posées à l'historiographie et par elle (id., p. 22). 
Si la production est bien le principe de la recherche historienne, dans la mesure où elle saisit tout document comme le symptôme de ce qui l'a produit (id., p. 19), alors il est symptomatique du discours même de de Certeau de mesurer sa gêne conceptuelle à vouloir naviguer entre les concepts opposés de travail productif et improductif. On pourrait y lire l'impossibilité de sortir, même pour un aussi fin penseur, de la référence omniprésente à la production. Malgré donc ses distances prises avec la pratique aveugle de l'historiographie, de Certeau ne pourrait faire autrement que marquer sa dette - même oubliée - envers la production. A moins que, en essayant de tenir le pari d'un discours historique qui relèverait d'un travail productif, tout en avouant sa légitime appartenance au travail improductif, leur distinction (du coup conceptuelle et sociale) soit en quelque sorte levée. La question ne concernerait plus alors la relevance d'un discours, ce dont elle relève voire sa redevance, si l'on veut s'exprimer dans le lexique économique: la relevance d'un discours étant ce que ce discours paye à l'institution qui le légitime - la redevance, elle, étant un autre nom de la dette -, donc la question concernerait moins la relevance d'un discours que sa simple levée, sa capacité à prendre consistance, exactement comme, sous la réaction chimique des levures à la chaleur, une pâte lève. Il ne s'agirait donc pas, d'un geste hegelien, de relever l'opposition entre travail productif et travail improductif - - pas question dès lors d'une intervention de la médiation -, l'enjeu tiendrait plutôt à l'immédiateté de la levée de l'opposition (comme on parle d'une levée d'écrou pour des prisonniers qu'on libère).

Si l'on repense au phénomène de l'ex-duction: se donner à soi-même une dette envers une altérité radicale que l'on a inventée afin de s'empêcher de produire du nouveau à l'intérieur de la communauté sinon sous la forme d'une réception qui fait croître encore la dette, et ainsi de suite; ou à celui de la pro-duction: intérioriser la dette comme une série de contraintes sociales sous la double forme d'un oubli et d'un pouvoir afin de pouvoir produire constamment du nouveau aussitôt rejeté dans le passé et oublié, et ainsi de suite; on s'aperçoit que la portée de ces gestes demeure l'instauration de médiations. La dette est avant tout le désir de la médiation - autrement dit, et dans les deux cas, l'oubli de l'immédiateté. Mais l'immédiateté de quoi? Non pas du don, comme on pourrait le croire en cherchant à opposer don et production, car le don ne fait que nous offrir l'immédiateté dans son mouvement, de même que le travail produit l'espace de la médiation. A noter d'un 
côté cette permanente mémoire des ancêtres qui fonde toutes les pratiques sociales et autorise la dette, et de l'autre cette constante inscription de l'histoire qui se légitime dans le passé pour mieux l'enterrer; à relever par ailleurs la nécessaire dépense de temps dans l'oisiveté afin de mieux savoir en user, ou la toute aussi vitale épargne du temps dans le travail afin de mieux savoir en produire; on saisit peut-être ce qui motive cet oubli, voire cette peur de l'immédiateté: l'immédiat est à chaque fois ce qui nous met face à la mort. Peut-être n'est-il d'autre immédiateté que la mort.

Ôter, même partiellement, à l'histoire sa faculté de médiation ne saurait être évident. En s'établissant à partir d'une double altération temporelle (rendre le passé étranger au présent et rendre le passé étranger à lui-même), l'historiographie s'institue comme médiation du présent au passé. Pourtant il est un point qu'il faut souligner: dans ce double geste d'altération, l'histoire nous met aussi face à la mort, même si c'est pour mieux l'écarter. L'histoire fait sienne l'immédiateté de l'avoirlieu de la mort, de l'avoir-lieu comme mort, même si c'est pour le relever bien vite comme chimère, fantôme ou fable. À découvrir ainsi la mort en ce qu'elle offre d'immédiateté et d'évanouissement, l'histoire nous donne la chance de saisir, ou mieux, d'user de l'immédiat. C'est en effet ici que la notion de kairos peut acquérir une dimension pratique, et non le seul effet savant d'un vestige du passé ou d'une curiosité archaïque. Participer à l'art du kairos n'est pas chose évidente pour nous, en raison de notre sens de la temporalité. Nous ne prenons le temps que sous l'apprêt d'un bon calcul des chances, un opportunisme où l'occasion suppose toujours un but étranger, plus important, en tous les cas lointain, en vue duquel l'occasion a été saisie, maîtrisée, rentabilisée. Dans cette conception machiavélienne de l'opportunité, celle-ci ne fait office, en définitive, que de médiation dans un processus à plus longue échéance. Or le kairos grec certainement ne fut jamais cela, au moins pour la raison que l'insistance sur le processus est une injonction moderne[ 13 ]. Le kairos n'est pas un instant quelconque dans la suite indéfinie d'instants quelconques, tous homogènes et stables qui forment notre sentiment de la temporalité; c'est d'abord une valeur, que le juste usage des temps et des valeurs permet justement d'évaluer. Il participe donc d'une temporalité aux instants hétérogènes, non uniformes et instables. En outre il n'est pas au service d'un projet qui le dépasserait - même si l'on peut en user et permettre ainsi la réalisation d'un objet ou le succès d'une épreuve -, puisque la simple reconnaissance du kairos offre déjà la pleine saveur du 
succès. Le kairos apparaît d'autant plus comme une valeur qu'il se donne dans une impeccable totalité: il n'a aucun besoin du reste du temps. C'est bien en ce sens que se noue son lien au plaisir, en ce que le plaisir implique un certain rapport au temps.

Dans l'Éthique à Nicomaque, Aristote pense ainsi le plaisir ou la joie: tout mouvement a pour propriétés vitesse ou lenteur (...) Or au plaisir n'appartiennent ni l'une ni l'autre de ces sortes de mouvements. Il est assurément possible d'être amené vers le plaisir (esthenai: aoriste exprimant mouvement, kinesis, ou genèse, genesis) plus ou moins rapidement, comme aussi de se mettre en colère, mais on ne peut pas être dans l'état de plaisir (edesthai) rapidement, pas même par rapport à une autre personne, alors que nous pouvons marcher, croître, et ainsi de suite, plus ou moins rapidement. (...) En quel sens le plaisir serait-il un devenir? (1173 a 35). Le plaisir ne saurait donc être caractérisé en termes de vitesses ni de situations parallèles à d'autres actes ou à d'autre corps: le plaisir est seul, enclos en lui-même. Non qu'il ne puisse s'échanger, mais il demeure toujours égal à lui-même, sans gradation ni croissance ou décroissance. C'est pourquoi il ne relève pas du mouvement: ni au repos, ni mobile, il n'est le résultat d'aucun processus. Sans doute le fait d'entrer dans le plaisir requiert-il un saut, mais au moment où le saut se fait, le plaisir est là, parfait, impétueux et clos - une tempête dans un verre d'eau.

On admet d'ordinaire que l'acte de vision est parfait à n'importe quel moment de sa durée (car il n'a besoin d'aucun complément qui surviendrait plus tard et achèverait sa forme). Or telle semble bien être aussi la nature du plaisir: il est, en effet, un tout, et on ne saurait à aucun moment appréhender un plaisir dont la prolongation dans le temps conduirait la forme à sa perfection. C'est la raison pour laquelle il n'est pas non plus un mouvement. Tout mouvement, en effet, se déroule dans le temps, et en vue d'une certaine fin, comme par exemple dans le processus de construction d'une maison (...). Du plaisir, au contraire, la forme est parfaite à n'importe quel moment. On voit ainsi que plaisir et mouvement ne sauraient être que différents l'un de l'autre, et que le plaisir est au nombre de ces choses qui sont des touts parfaits. Cette conclusion pourrait aussi résulter du fait qu'il est impossible de se mouvoir autrement que dans le temps, alors qu'il est possible de ressentir le plaisir indépendamment du temps, car ce qui a lieu dans l'instant est un tout complet. (1174 a 15 - b 5) Le plaisir, comme tout acte, a bien lieu dans le temps, mais dans un rapport particulier 
au temps: il n'est pas discontinu, fragmentable, nombrable; à l'instar de la vue, il forme un tout parfait, achevé en tout moment de sa durée. Le plaisir a lieu dans le temps et hors du mouvement du temps. Il n'ouvre pas pour autant à l'éternité, forme de négation du temps, puisqu'il peut cesser à tout moment (non pas s'affaiblir doucement, paraître moins véloce dans la sensation qu'il nous donne, mais disparaître brusquement de la vue). L'expérience amoureuse est bien celle-là. Quand Swann un matin considère Odette, ne la trouvant pas moins jolie que la veille, ni moins désirable ou moins fugitive, voilà qu'il a le sentiment d'émerger d'une bulle de plaisir et de peine, ne pouvant plus que constater qu'il avait connu le grand amour de sa vie pour une femme qui n'était pas [son] type - ce genre de regard ne peut être porté que si la perspective a totalement changé et le plaisir définitivement disparu.

Le plaisir, ou la joie, s'oppose au processus. Il requiert complétude, totalité et surtout immédiateté. On ne peut rien lui ajouter, on ne peut en faire un empilage de médiations. Il n'existe donc jamais de dialectique du plaisir, seulement une monadologie de la joie. La joie vient de cette plénitude, cette totalité soudain offerte, sans que le temps, qu'il apparaisse sous le masque avare de l'épargne ou celui, prodigue, de la dépense, ait jamais prise sur elle - mais aussi c'est l'immédiateté elle-même qui fait la joie. La joie n'est pas hors du temps, elle ne lui est pas extérieure, et elle n'y est pas prise non plus. Elle n'appartient ni à l'éternité, dans la mesure où elle peut cesser, ni au temps, dans la mesure où elle n'est pas segmentable en unités de durée. La joie, au moment où elle illumine l'ombre portée des êtres et des choses, ne réclame aucun travail, ni productif, ni improductif (ce qui ne signifie pas qu'il ne puisse y avoir de travail effectué afin de se préparer à sa venue, de même que le sophiste s'exerce à l'advenue du kairos). Pas de recherche au sens d'une production, pas d'enseignement en charge de sa reproduction. Nul ne peut servir de médiation à la joie, sauf à l'ignorer. En rechercher et en guetter l'apparition, là est la seule manière de tourner la quête de savoir et son enseignement. Si la joie, en son immédiateté, n'est ni éternelle, ni temporelle, elle ne peut donc être qu'immortelle. L'immortalité n'est, à l'évidence, pas une affaire de durée, mais un sentiment de l'immédiateté; il ne s'agit pas d'être présent jusqu'à la fin des temps et au-delà; il ne s'agit pas non plus d'être voué à la fugacité du temps qui passe qu'on s'imagine maîtriser en le mesurant; l'immortalité tient toute entière en chaque instant où la figure messianique de la joie apparaît. Ni dans le repliage soumis du présent sur le passé, ni dans le dépliage laborieux du passé et du 
présent, mais dans la mince pliure des temps par où le muscle contracté du passé se dilate dans le présent.

On pourrait penser que nous arrivons là à une conclusion rigoureusement inverse de celle qui nous avait conduit, auparavant, de l'immédiateté à la mort. Inverse, certes; pas contradictoire. C'est bien parce qu'il n'est d'immédiateté que de la mort, que la joie, comme sentiment de l'immédiat, nous apporte l'immortalité. Mort et joie sont les deux faces d'une monnaie avec laquelle nous payons nos dettes. Si, de la fin d'un artiste du kairos comme Socrate, il nous est possible d'apprendre quelque chose, ce ne peut être que cette joie de mourir dont il fait preuve. Tout le Phédon est un discours, devant la mort, de l'immortalité de l'âme. Les mythes auxquels Socrate recourt ne lui font pas illusion: il ne faudrait pas avoir son bon sens pour y croire littéralement (ce ne sont que des médiations de l'immédiateté). Mais ils témoignent de la présence de l'immortalité en celui qui prend le risque d'y reconnaître la vertu de son âme: ce risque (kindunos : le danger, le péril) est beau en effet, et dans des croyances de cette sorte il y a comme une incantation (epado, c'est accompagner par un chant, mais aussi user de charmes!) qu'il faut se faire à soi-même (Phédon, $114 \mathrm{~d}$ ). La joie est l'incantation de l'immortalité, ce par où la mort chante. Même si, pour Socrate, l'harmonie en est plus belle une fois passé le lac Achérousias, une fois libéré de la geôle du corps, cette musique, avant de mourir, est déjà nôtre. Certes nous ne vivons que dans un univers de médiations soigneusement construites par nous-mêmes, comme pour mieux donner à l'immédiateté sa plus fascinante figure, à moins que ce ne soit pour raffermir encore notre peur à son égard - mais n'est-ce pas le même geste? C'est encore et toujours par la médiation du discours, qu'il soit dialogue platonicien ou historiographie, que la joie de mourir peut nous apparaître. Les ruses de la médiation sont infinies pour prétendre à ce qu'elle n'est pas. Ainsi de ce texte même qui tente, dans le miroir d'une dérive sur les eaux sombres du savoir, d'apercevoir la clarté d'un ami disparu. Si la force de sa présence se maintient, cela tient certainement à sa manière d'avoir tourné, avec obstination, plaisirs du corps et plaisirs du savoir en incantation joyeuse. À nous la tâche d'y répondre.

"Eh bien! dit Criton, quels ordres nous donnes-tu, Socrate, à ceux-ci ou à moi, soit au sujet de tes enfants, soit pour toute autre affaire? De notre part cette tâche 
serait, par amour pour toi, notre tâche principale! Justement, Criton, je ne cesse pas d'en parler, réponditil, et il n'y a rien de neuf à en dire! Voici: ayez, vous, le souci de vous-mêmes, et de votre part alors toute tâche sera une tâche faite par amour, et pour moi, et pour ce qui est mien, et pour vous-mêmes, n'eussiez-vous à présent pas pris d'engagement! (...) - Nous mettrons, c'est entendu, tout notre coeur, dit Criton, à nous conduire ainsi. Mais tes funérailles, comment y procèderons-nous? - Comme il vous plaira, répondit-il; à condition bien sûr que vous mettiez la main sur moi et que je ne vous échappe pas!" Là-dessus, il se mit à rire doucement (Phédon, 115 b-c).

\section{NOTES}

1. Platon, Phédon, trad. L. Robin, Paris, Les Belles Lettres, 1965, 118 a.

2. "The University becomes modern when it takes on responsibility for working out the relation between the subject and the state, when it offers to incarnate an Idea that will both theorise and inculcate this relationship. This is its dual mission of research and teaching", Bill Readings, "For a Heteronomous Cultural Politics : The University, Culture and the State", Oxford Literary Review, vol. 15, $\mathrm{n}^{\circ} 1-2,1993, \mathrm{p} .171$.

3. Wlad Godzich, "Afterword", in Samuel Weber, Institution and Interpretation, Minneapolis, Minnesota Press, 1987, p. 161.

4. Sur les liens entre esthétique du sublime et dette, voir l'ouvrage de Peter de Bolla, The Discourse of the Sublime, London, Blackwell, 1989.

5. Adam Smith, An Inquiry into the Nature and Causes of the Wealth of Nations, ed. E. Cannan, University of Chicago Press, 1976. Il faut noter que l'origine de l'opposition productif/improductif se trouve dans les théories des physiocrates; pour eux, la nature seule est vraie source de richesse et le travail des hommes ne saurait que la "féconder". Par conséquent le travail véritablement producteur de richesses ne sera que celui des travailleurs de la terre. Tous les autres ne font que transformer les produits des "classes productives" et sont considérés comme "stériles". Adam Smith reprend 
le principe de l'opposition production/stérilité, mais en efface le vocabulaire et la référence naturalistes en fondant son concept de travail sur l'échange impliqué par la division sociale du travail. Ce faisant il annihile l'ultime vestige de l'ancienne conception de la production, son caractère de force naturelle.

6. Jean-Pierre Vernant, Pierre Vidal-Naquet, Travail et esclavage en Grèce ancienne, Paris, Ed. Complexe, 1988, p. 33.

7. Voir Jacques Le Goff, Pour un autre Moyen Age, Paris, Gallimard, 1977.

8. Rousseau, Essai sur l'origine des langues, Paris, Bibliothèque du Graphe, 1969, p. 521.

9. Pas seulement d'ailleurs l'économie politique puisque le concept de travail trouve justement sa formulation en physique autour de 1800. Voir sur ce point François Vatin, Le travail : Economie et physique, 1780-1830, PUF, 1993.

10. Sur cette importance accordée au processus, Hannah Arendt note que les théoriciens politiques à partir du XVIIe siècle furent en présence d'un processus inouï d'accroissement de richesse, de propriété, d'acquisition. En essayant d'expliquer cette augmentation constante, ils remarquèrent naturellement le phénomène du processus progressif, de sorte que (...) le concept de processus devint le concept-clef de l'époque et de ses sciences, historiques et naturelles. Dès le début, on se représenta ce processus, puisqu'il paraissait sans fin, comme un processus naturel et plus spécialement sous l'aspect du processus vital. (...) De toutes les activités humaines seul le travail (ni l'action ni l'oeuvre) ne prend jamais fin, et avance automatiquement d'accord avec la vie, Condition de l'homme moderne, Presses Pocket, 1983, p. 152.

11. Cité par Reinhart Koselleck, Vergangene Zukunft : Zur Semantik geschichtlicher Zeiten, Frankfurt am Main, Surhkamp, 1979.

12. Michel de Certeau, L'écriture de l'histoire, Gallimard, 1975, p. 9.

13. Hannah Arendt : À la place du concept d'Être nous trouvons maintenant le concept de processus. Et tandis que c'est le propre de l'Être d'apparaître et ainsi de se dévoiler, c'est le propre du processus de demeurer invisible, de rester quelque chose dont on peut seulement inférer l'existence d'après la présence de 
certains phénomènes. Ce processus fut à l'origine celui de la fabrication qui "disparaît dans le produit", et il se fondait sur l'expérience de l'homo faber, qui savait qu'un processus de production précède nécessairement l'existence de tout objet. , La condition de l'homme moderne, Presses Pocket, 1994 (1958), p. 371.

Accueil Surfaces | Table des matières | Recherche $\underline{\text { Surfaces Home Page | Table of Contents | Search }}$

PUM | Livres | Revues | Publications électroniques | Vente et distribution 